\title{
Distributive equation of implications based on continuous triangular norms
}

\author{
Feng Qin, ${ }^{1,2}$ Michał Baczyński* ${ }^{3}$ Aifang Xie ${ }^{4}$ \\ ${ }^{1}$ College of Mathematics and Information Science, Nanchang Hangkong University, 330063, Nanchang, P.R. China \\ ${ }^{2}$ College of Mathematics and Information Science, Jiangxi Normal University, 330022, Nanchang, P.R. China \\ ${ }^{3}$ Institute of Mathematics, University of Silesia, ul. Bankowa 14, 40-007 Katowice, Poland \\ ${ }^{4}$ School of Mathematics, Shandong University, 250100, Jinan, P.R. China
}

\begin{abstract}
In order to avoid combinatorial rule explosion in fuzzy reasoning, in this work we explore the distributive equations of implications. In details, by means of the section of $I$, we give out the sufficient and necessary conditions of solutions for the distributive equation of implication $I\left(x, T_{1}(y, z)\right)=$ $T_{2}(I(x, y), I(x, z))$, when $T_{1}$ is a continuous but not Archimedean triangular norm, $T_{2}$ is a continuous Archimedean triangular norm and $I$ is an unknown function. Our methods of proof can be applied to the three other functional equations related closely to the distributive equation of implication.
\end{abstract}

Keywords: Combs methods, functional equations, fuzzy implication, t-norm, continuous t-norm.

\section{Introduction}

The ability to build complex commercial and scientific fuzzy logic applications has been hampered by what is popularly known as the combinatorial rule explosion problem, which is associated with the conventional fuzzy rule configuration and its accompanying rule matrix. Since all the rules of an inference engine are exercised during every inference cycle, the number of rules directly affects the computational duration of the overall application. To reduce complexity of fuzzy "IF-THEN" rules, Combs and Andrews [7] required of the following classical tautology

$$
(p \wedge q) \rightarrow r \equiv(p \rightarrow r) \vee(q \rightarrow r) .
$$

They refer to the left-hand side of this equivalence as an intersection rule configuration (IRC) and to its right-hand side as a union rule configuration (URC). Subsequently, there were many discussions (see [10], [15], [8], [9]), most of them pointed out the need for a theoretical investigation required for employing such equations, as concluded by Dick and Kandel [10], "Future work on this issue will require an examination of the properties of various combinations of fuzzy unions, intersections and implications" or by Mendel and Liang [15], "We think that what this all means is that we have to

\footnotetext{
${ }^{*}$ Corresponding author
}

look past the mathematics of IRC $\Leftrightarrow \mathrm{URC}$ and inquire whether what we are doing when we replace IRC by URC makes sense." And then, Trillas and Alsina [20], in the standard fuzzy theory, turned the about requirement into the functional equation $I(T(x, y), z)=S(I(x, z), I(y, z))$ and obtained all solutions of $T$ and $S$ when $I$ are special cases of $R$ implications, $S$-implications and $Q L$-implications, respectively. Along the lines, Balasubramaniam and Rao [6] investigated the other three functional equations interrelated with this equation. In order to study it in more general case, Ruiz-Aguilera and Torrens [18], [19] and Qin and Zhao [17], in their own papers, generalized the above equation into uninorms.

On the other hand, from fuzzy logical angle, Türksen et al. [21] posed and discussed the equation

$$
I(x, T(y, z))=T(I(x, y), I(x, z)),
$$

where $x, y, z \in[0,1]$, and then, got the necessary conditions for a fuzzy implication $I$ to satisfy Eq. (1) when $T$ is the product t-norm. Later, Baczyński [1] generalized some Türksen's results into strict t-norms and obtained the sufficient and necessary conditions of functional equations consisting of Eq. (1) and the following equation

$$
I(x, I(y, z))=I(T(x, y), z), \quad x, y, z \in[0,1] .
$$

Moreover, he (see [2]) also tried to give out the sufficient and necessary conditions of the functional equations composed of Eq. (1) and the following equation

$$
I(x, y)=I(N(y), N(x)), \quad x, y, z \in[0,1] .
$$

Unfortunately, his results only apply for a particular case. After this, Yang and Qin in [22] got their full characterizations. Subsequently, many people [2], [5], [16], including Baczyński and Qin, investigate again the distributivity of fuzzy implications over nilpotent (strict) triangular norms (conorms). Rather recently, as for the functional equation $I\left(x, S_{1}(y, z)\right)=S_{2}(I(x, y), I(x, z))$, which is one of the three other functional equations related closely to the following Eq. (D), Baczyński and Balasubramaniam in [5] pointed out "the situation when $S_{1}$ and $S_{2}$ are continuous t-conorms is still unsolved." 
In this paper, we explore only the following distributive equation of implication,

$$
I\left(x, T_{1}(y, z)\right)=T_{2}(I(x, y), I(x, z)),
$$

where $x, y, z \in[0,1]$ and when $T_{1}$ is a continuous but not Archimedean triangular norm, $T_{2}$ is a continuous, Archimedean triangular norm and $I$ is an unknown function. But our methods can still apply to the three other functional equations related closely to Eq. (D). It is in this sense that we make progress in solving the functional equation $I\left(x, S_{1}(y, z)\right)=S_{2}(I(x, y), I(x, z))$ suggested by Baczyński and Balasubramaniam in [5].

\section{Preliminaries}

In this section, we recall basic notations and facts used in the sequel.

Definition 2.1 ([13], [12]). (i) A binary function $T:[0,1]^{2} \rightarrow[0,1]$ is called a t-norm, if it is increasing in both variables, commutative, associative and has 1 as the neutral element.

(ii) A t-norm $T$ is said to be Archimedean if for every $x, y \in(0,1)$ there exists $n \in \mathbb{N}$ such that $x_{T}^{[n]}<y$, where

$$
x_{T}^{[n]}:= \begin{cases}x, & n=1 \\ T\left(x, x_{T}^{[n-1]}\right), & n>1 .\end{cases}
$$

(iii) A t-norm $T$ is said to be strict, if $T$ is continuous and strictly monotone, i.e., $T(x, y)<$ $T(x, z)$ whenever $x \in(0,1]$ and $y<z$.

(iv) A t-norm $T$ is said to be nilpotent, if it is continuous and if each $x \in(0,1)$ is a nilpotent element of $T$, i.e., if there exists $n \in \mathbb{N}$ such that $x_{T}^{[n]}=0$.

Remark 2.2 (see [13], [12]). (i) A continuous t-norm $T$ is Archimedean if and only if it holds $T(x, x)<x$ for all $x \in(0,1)$.

(ii) If $T$ is strict or nilpotent, then it must be Archimedean. The converse is also true when it is continuous.

We shall use the following characterizations of continuous (Archimedean) t-norms.

Theorem 2.3 ([14], cf. [13, Theorem 5.1]). For a function $T:[0,1]^{2} \rightarrow[0,1]$ the following statements are equivalent:

(i) $T$ is a continuous, Archimedean t-norm.

(ii) $T$ has a continuous additive generator, i.e., there exists a continuous, strictly decreasing function $t:[0,1] \rightarrow[0, \infty]$ with $t(1)=0$, which is uniquely determined up to a positive multiplicative constant, such that

$$
T(x, y)=t^{-1}(\min (t(x)+t(y), t(0))),
$$

for all $x, y \in[0,1]$
Remark 2.4 ([13, Section 3.2]). (i) $T$ is a strict t-norm if and only if each continuous additive generator $t$ of $T$ satisfies $t(0)=\infty$.

(ii) $T$ is a nilpotent t-norm if and only if each continuous additive generator $t$ of $T$ satisfies $t(0)<\infty$.

Theorem 2.5 ([13, Theorem 5.11]). For a function $T:[0,1]^{2} \rightarrow[0,1]$ the following statements are equivalent:

(i) $T$ is a continuous t-norm.

(ii) $T$ is uniquely representable as an ordinal sum of continuous Archimedean t-norms, i.e., there exists a uniquely determined (finite or countable infinite) index set A, a family of uniquely determined pairwise non-overlapping, closed, proper subintervals $\left(\left[a_{m}, b_{m}\right]\right)_{m \in A}$ of $[0,1]$ and a family of uniquely determined continuous Archimedean t-norm $\left(T_{m}\right)_{m \in A}$ such that

$$
T(x, y)=\left\{\begin{array}{c}
a_{m}+\left(b_{m}-a_{m}\right) T_{m}\left(\frac{x-a_{m}}{b_{m}-a_{m}} \frac{y-a_{m}}{b_{m}-a_{m}}\right) \\
\quad(x, y) \in\left[a_{m}, b_{m}\right]^{2} \\
\min (x, y), \quad \text { otherwise. }
\end{array}\right.
$$

We shall write $\left.T=\left(<a_{m}, b_{m}, T_{m}\right\rangle\right)_{m \in A}$.

Observe that if $A=\emptyset$, then $T$ is the minimum t-norm $T_{\mathbf{M}}$, while if $\operatorname{card} A=1$ and $a_{1}=0$ and $b_{1}=1$, then $T$ is a continuous Archimedean t-norm, i.e., it is strict or nilpotent.

Finally, let us recall the definition of a fuzzy implication. In the literature we can find several diverse definitions of fuzzy implications. In this article we will use the following one, which is equivalent to the definition used by Fodor and Roubens [11, Definition 1.15] (see also Baczyński and Jayaram [4]).

Definition 2.6. A function $I:[0,1]^{2} \rightarrow[0,1]$ is called a fuzzy implication if it satisfies the following conditions:

$I$ is decreasing in the first variable,

$I$ is increasing in the second variable,

$$
I(0,0)=I(1,1)=I(0,1)=1, \quad I(1,0)=0 .
$$

\section{First solutions to Eq. (D) when $T_{1}$ is a continuous t-norm}

In this section, we present first characterizations of function $I$ satisfying Eq. (D) when $T_{1}$ is a continuous t-norm and $T_{2}$ is a strict t-norm. Unless otherwise stated, we always assume that $T_{1}$ is only continuous but not Archimedean, which means that $T_{1}$ must have at least one non-trivial idempotent element, because the case that $T_{1}$ is continuous and Archimedean has been studied by Baczyński [1], [2], [5], Qin [16], [22], and so on. 
Lemma 3.1. Let $T_{1}$ be a continuous t-norm, $T_{2}$ be a continuous Archimedean t-norm and $I:[0,1]^{2} \rightarrow$ $[0,1]$ be a binary function. If the triple of functions $T_{1}, T_{2}, I$ satisfies Eq. (D) for every $x, y, z \in[0,1]$, then either $I(x, y)=0$ or $I(x, y)=1$ hold for every $x \in[0,1]$ and every $y \notin \cup_{m \in A}\left(a_{m}, b_{m}\right)$, i.e., $y$ is an idempotent element of $T_{1}$.

Proof. Suppose that $y \notin \cup_{m \in A}\left(a_{m}, b_{m}\right)$, i.e., $y$ is an idempotent element of $T_{1}$. Then we know that $T_{1}(y, y)=y$ and it follows from Eq. (D) that

$$
I(x, y)=T_{2}(I(x, y), I(x, y)), \quad x \in[0,1],
$$

which means that the value of $I(x, y)$ is also an idempotent element of $T_{2}$. Note that $T_{2}$ is Archimedean and thus has only two trivial idempotent elements 0 or 1 . Hence either $I(x, y)=0$ or $I(x, y)=1$ hold for all $x \in[0,1]$.

Lemma 3.2. Let $T_{1}$ be a continuous t-norm, $T_{2}$ be a continuous Archimedean t-norm, $I:[0,1]^{2} \rightarrow$ $[0,1]$ be a binary function and $y_{0}$ be a fixed idempotent element of $T_{1}$. If the triple of functions $T_{1}, T_{2}, I$ satisfies Eq. (D) for all $x, y, z \in[0,1]$, then

(i) if it holds $I\left(x, y_{0}\right)=0$ for some $x \in[0,1]$, then it follows $I(x, z)=0$ for any $z \leq y_{0}$;

(ii) if it holds $I\left(x, y_{0}\right)=1$ for some $x \in[0,1]$, then it follows $I(x, z)=1$ for any $z \geq y_{0}$.

Proof. We only prove the case (i) because the case (ii) is similar. Note that $y_{0}$ is an idempotent element of $T_{1}$, then we obtain that $T_{1}\left(y_{0}, z\right)=\min \left(y_{0}, z\right)$ for any $z \in[0,1]$. By virtue of our assumption $z \leq y_{0}$ it holds, from Eq. (D), that

$$
I(x, z)=T_{2}\left(I\left(x, y_{0}\right), I(x, z)\right) .
$$

Further, applying the hypothesis $I\left(x, y_{0}\right)=0$, we get that $I(x, z)=0$ for any $z \leq y_{0}$.

According to the above analysis, we have the following result.

Lemma 3.3. Let $T_{1}$ be a continuous t-norm, $T_{2}$ be a continuous Archimedean t-norm, $I:[0,1]^{2} \rightarrow$ $[0,1]$ be a binary function and $y_{1}, y_{2}$ be two different idempotent elements of $T_{1}$. Assume that the triple of functions $T_{1}, T_{2}, I$ satisfies Eq. (D) for all $x, y, z \in[0,1]$. If both $I\left(x, y_{1}\right)=0$ and $I\left(x, y_{2}\right)=1$ are simultaneously true for some $x \in[0,1]$, then we must have $y_{1}<y_{2}$.

Proof. On the contrary, suppose $y_{1}>y_{2}$ and then take $z \in\left(y_{2}, y_{1}\right)$. On one hand, applying case (i) in Lemma 3.2 , we can know that $I(x, z)=0$. On the other hand, applying case (ii) in Lemma 3.2, we can know that $I(x, z)=1$. This is a contradiction.

For a given binary function $I$ and fixed $x \in[0,1]$, we define

$$
\begin{aligned}
U_{(I, x)}=\{y \in[0,1] \mid I(x, y)=0 \text { and } \\
\left.y \text { is an idempotent element of } T_{1}\right\}, \\
u_{(I, x)}=\sup U_{(I, x)},
\end{aligned}
$$

and

$$
\begin{aligned}
V_{(I, x)}= & \{y \in[0,1] \mid I(x, y)=1 \text { and } \\
& \left.\quad y \text { is an idempotent element of } T_{1}\right\}, \\
v_{(I, x)}= & \inf V_{(I, x)} .
\end{aligned}
$$

We stipulate here that $\sup \emptyset=0$ and $\inf \emptyset=1$. By Lemma 3.3, we have $u_{(I, x)} \leq v_{(I, x)}$. Since $T_{1}$ is continuous and monotone, it follows that both $u_{(I, x)}$ and $v_{(I, x)}$ are idempotent elements of $T_{1}$, too. Now, by the order between $u_{(I, x)}$ and $v_{(I, x)}$, we have to consider two cases: $u_{(I, x)}=v_{(I, x)}$ and $u_{(I, x)}<$ $v_{(I, x)}$.

Using previous lemmas one can obtain the following first main result.

Theorem 3.4. Let $T_{1}$ be a continuous t-norm, $T_{2}$ be a continuous Archimedean t-norm, I: $[0,1]^{2} \rightarrow$ $[0,1]$ be a binary function and assume that $u_{(I, x)}=$ $v_{(I, x)}$ for some fixed $x \in[0,1]$. Then the following statements are equivalent:

(i) The triple of functions $T_{1}, T_{2}, I(x, \cdot)$ satisfies $E q$. (D) for any $y, z \in[0,1]$.

(ii) The vertical section $I(x, \cdot)$ has one of the following forms:

(a) If $\mu_{(I, x)} \in U_{(I, x)}$, then

$$
I(x, y)= \begin{cases}0, & y \leq \mu_{(I, x)}, \\ 1, & y>\mu_{(I, x)}\end{cases}
$$

for all $y \in[0,1]$.

(b) If $\nu_{(I, x)} \in V_{(I, x)}$, then

$$
I(x, y)= \begin{cases}0, & y<\nu_{(I, x)}, \\ 1, & y \geq \nu_{(I, x)}\end{cases}
$$

for all $y \in[0,1]$.

Example 3.5. Assume that $T_{1}$ is a continuous t-norm given by the ordinal sum

$$
T=\left(\left\langle 0.1,0.5, T_{\mathbf{P}}\right\rangle,\left\langle 0.7,0.9, T_{\mathbf{L K}}\right\rangle\right),
$$

where $T_{\mathbf{P}}(x, y)=x y$ is the product t-norm and $T_{\mathbf{L K}}(x, y)=\max (x+y-1,0)$ is the Eukasiewicz t-norm. Thus we have

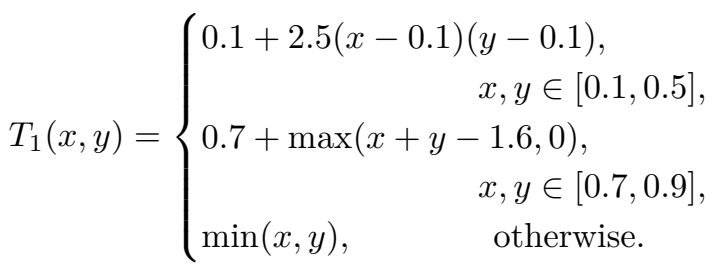

The set of idempotent elements for $T_{1}$ is the following:

$$
\operatorname{Idem}\left(T_{1}\right)=[0,0.1] \cup[0.5,0.7] \cup[0.9,1] .
$$

One can easily check that the following functions $I$ satisfy Eq. (D) (for any $x, y, z \in[0,1]$ ) with $T_{1}$ and any continuous Archimedean t-norm $T_{2}$ : 
- $I=0$; in this case $u_{(I, x)}=v_{(I, x)}=1$ for any $x \in[0,1]$ and $I$ has the form (4).

- $I=1$; in this case $u_{(I, x)}=v_{(I, x)}=0$ for any $x \in[0,1]$ and $I$ has the form (5).

- $I(x, y)=\left\{\begin{array}{ll}0, & y \leq 0.6, \\ 1, & y>0.6,\end{array} ;\right.$ in this case $u_{(I, x)}=$ $v_{(I, x)}=0.6$ for any $x \in[0,1]$ and $I$ has the form (4).

- $I(x, y)=\left\{\begin{array}{ll}0, & y<0.6, \\ 1, & y \geq 0.6,\end{array} ;\right.$ in this case $u_{(I, x)}=$ $v_{(I, x)}=0.6$ for any $x \in[0,1]$ and $I$ has the form (5).

Corollary 3.6. Let $T_{1}=T_{\mathrm{M}}, T_{2}$ be a continuous Archimedean t-norm and $I:[0,1]^{2} \rightarrow[0,1]$ be a binary function. Then the following statements are equivalent:

(i) The triple of functions $T_{\mathrm{M}}, T_{2}, I$ satisfies $E q$. (D) for any $x, y, z \in[0,1]$.

(ii) For any $x \in[0,1]$ there exist a constant $c_{x} \in$ $[0,1]$ such that the vertical section $I(x, \cdot)$ has one of the following forms:

$$
\begin{aligned}
& I(x, y)=\left\{\begin{array}{ll}
0, & y \leq c_{x}, \\
1, & y>c_{x},
\end{array} \quad y \in[0,1],\right. \\
& I(x, y)=\left\{\begin{array}{ll}
0, & y<c_{x}, \\
1, & y \geq c_{x},
\end{array} \quad y \in[0,1] .\right.
\end{aligned}
$$

Now, let us consider the case $u_{(I, x)}<v_{(I, x)}$.

Lemma 3.7. Let $T_{1}$ be a continuous t-norm, $T_{2}$ be a continuous Archimedean t-norm, I: $[0,1]^{2} \rightarrow$ $[0,1]$ be a binary function and fix $x \in[0,1]$. If $u_{(I, x)}<v_{(I, x)}$ and the triple of functions $T_{1}, T_{2}, I(x, \cdot)$ satisfies Eq. (D) for all $y, z \in$ $[0,1]$, then there exists some $m_{0} \in A$ such that $\left[u_{(I, x)}, v_{(I, x)}\right]=\left[a_{m_{0}}, b_{m_{0}}\right]$, where $A$ and $\left[a_{m_{0}}, b_{m_{0}}\right]$ are the index set and the subinterval in Theorem 2.5, respectively.

Proof. Let us fix arbitrarily $x \in[0,1]$. If there exists an idempotent element $c$ such that $c \in$ $\left(u_{(I, x)}, v_{(I, x)}\right)$, then, according to Lemma 3.1, we get that either $I(x, c)=0$ or $I(x, c)=1$. If $I(x, c)=0$, then this is a contradiction with the definition of $u_{(I, x)}$. If $I(x, c)=1$, then this is a contradiction with the definition of $v_{(I, x)}$. This means that there are no idempotent elements between $u_{(I, x)}$ and $v_{(I, x)}$. Note that both $u_{(I, x)}$ and $v_{(I, x)}$ themselves are idempotent and different, hence it follows that there exists some $m_{0} \in A$ such that $\left[u_{(I, x)}, v_{(I, x)}\right]=\left[a_{m_{0}}, b_{m_{0}}\right]$.

So far, we have proven the fact that

$$
I(x, y)= \begin{cases}0, & y<u_{(I, x)} \\ 1, & y>v_{(I, x)}\end{cases}
$$

for any fixed $x \in[0,1]$, when $u_{(I, x)}<v_{(I, x)}$. But we do say nothing about the values of $I(x, y)$ for any $y \in\left[u_{(I, x)}, v_{(I, x)}\right]$. We will solve this problem in next sections.

\section{Solutions to Eq. (D) when $T_{1}$ is a continuous t-norm and $T_{2}$ is a strict t-norm}

Using the solutions of the additive Cauchy functional equation

$$
f(x+y)=f(x)+f(y),
$$

when $x, y \in[0, \infty]$ and $f:[0, \infty] \rightarrow[0, \infty](\operatorname{see}[5$, Proposition 2]) we are able to prove the following theorem.

Theorem 4.1. Let $T_{1}$ be a continuous t-norm, $T_{2}$ be a strict $t$-norm, $I:[0,1]^{2} \rightarrow[0,1]$ be a binary function and fix arbitrarily $x \in[0,1]$. If $u_{(I, x)}<u_{(I, x)}$ and the correspondingly generating t-norm $T_{m_{0}}$ of $T_{1}$ on the generating subinterval $\left[u_{(I, x)}, v_{(I, x)}\right]=\left[a_{m_{0}}, b_{m_{0}}\right]$ is strict, then the following statements are equivalent:

(i) The triple of functions $T_{1}, T_{2}, I(x, \cdot)$ satisfies $E q$. (D) for all $y, z \in[0,1]$.

(ii) $T_{1}$ admits the representation (3), and there exist continuous, strictly decreasing functions $t_{m_{0}}, t_{2}:[0,1] \rightarrow[0, \infty]$ with $t_{m_{0}}(1)=t_{2}(1)=$ $0, t_{m_{0}}(0)=t_{2}(0)=\infty$, which are uniquely determined up to positive multiplicative constants, such that the correspondingly generating t-norm $T_{m_{0}}$ of $T_{1}$ on the generating subinterval $\left[a_{m_{0}}, b_{m_{0}}\right]$ and $T_{2}$ admit the representation (2) with $t_{m_{0}}, t_{2}$, respectively, and for the fixed above $x \in[0,1]$, the vertical section $I(x, \cdot)$ has one of the following forms:

$$
\begin{aligned}
& I(x, y)= \begin{cases}0, & y \in\left[0, a_{m_{0}}\right], \\
1, & y \in\left(a_{m_{0}}, 1\right],\end{cases} \\
& I(x, y)= \begin{cases}0, & y \in\left[0, b_{m_{0}}\right), \\
1, & y \in\left[b_{m_{0}}, 1\right],\end{cases}
\end{aligned}
$$

$I(x, y)= \begin{cases}0, & y \in\left[0, a_{m_{0}}\right], \\ t_{2}^{-1}\left(c_{x} t_{m_{0}}\left(\frac{y-a_{m_{0}}}{b_{m_{0}}-a_{m_{0}}}\right)\right), & y \in\left[a_{m_{0}}, b_{m_{0}}\right], \\ 1, & y \in\left[b_{m_{0}}, 1\right],\end{cases}$

with a certain $c_{x} \in(0, \infty)$, uniquely determined up to a positive multiplicative constant depending on constants for $t_{m_{0}}$ and $t_{2}$.

Example 4.2. Let us consider the same continuous t-norm as in Example 3.5:

$$
T=\left(\left\langle 0.1,0.5, T_{\mathbf{P}}\right\rangle,\left\langle 0.7,0.9, T_{\mathbf{L K}}\right\rangle\right) .
$$

Let us assume that for any $x \in[0,1]$ we have $u_{(I, x)}=0.1$ and $v_{(I, x)}=0.5$. This means that $T_{m_{0}}=T_{\mathbf{P}}$ is a strict t-norm and $t_{m_{0}}(x)=-\ln x$. Let us assume that also $T_{2}(x, y)=x y$ i.e., $t_{2}(x)=$ $-\ln x$. One can easily check that the following functions $I$ satisfy Eq. (D) (for any $x, y, z \in[0,1]$ ) with $T_{1}$ and $T_{2}$ :

- $I(x, y)= \begin{cases}0, & y \in[0,0.1] \\ 1, & y \in(0.1,1]\end{cases}$ 


$$
\begin{aligned}
& \text { - } I(x, y)= \begin{cases}0, & y \in[0,0.5), \\
1, & y \in[0.5,1],\end{cases} \\
& \text { - } I(x, y)= \begin{cases}0, & y \in[0,0.1], \\
\frac{y-0.1}{0.4}, & y \in[0.1,0.5], \\
1, & y \in[0.5,1] .\end{cases}
\end{aligned}
$$

Observe also that idempotent elements are really crucial in the above theorem. For example the following function

$$
I(x, y)= \begin{cases}0, & y \in[0,0.3], \\ 1, & y \in(0.3,1],\end{cases}
$$

does not satisfy Eq. (D) with $T_{1}$ and $T_{2}$. Indeed, for any $x \in[0,1]$ we have

$$
I\left(x, T_{1}(0.36,0.36)\right)=I(x, 0.269)=0,
$$

while

$$
T_{2}(I(x, 0.36), I(x, 0.36))=T_{2}(1,1)=1 .
$$

Example 4.3. Assume that $T_{1}$ is a continuous t-norm given by the ordinal sum

$$
T_{1}=\left(\left\langle 0.25,0.75, T_{\mathbf{P}}\right\rangle,\left\langle 0.75,1, T_{\mathbf{L K}}\right\rangle\right),
$$

thus

$$
T_{1}(x, y)=\left\{\begin{array}{r}
0.25+2(x-0.25)(y-0.25), \\
x, y \in[0.25,0.75], \\
0.75+\max (x+y-1.75,0), \\
x, y \in[0.75,1], \\
\min (x, y), \quad \text { otherwise }
\end{array}\right.
$$

The set of idempotent elements for $T_{1}$ is the following:

$$
\operatorname{Idem}\left(T_{1}\right)=[0,0.25] \cup\{0.75,1\} .
$$

Let us assume that for any $x \in[0,1]$ we have $u_{(I, x)}=0.25$ and $v_{(I, x)}=0.75$. This means that $T_{m_{0}}=T_{\mathbf{P}}$ is a strict t-norm and $t_{m_{0}}(x)=-\ln x$. One can easily check that the following functions $I$ satisfy Eq. (D) with $T_{1}$ and the product t-norm $T_{\mathbf{P}}$ (for any $x, y, z \in[0,1])$ :

$$
\begin{aligned}
& I(x, y)= \begin{cases}0, & y \in[0,0.25], \\
1, & y \in(0.25,1],\end{cases} \\
& I(x, y)= \begin{cases}0, & y \in[0,0.75), \\
1, & y \in[0.75,1],\end{cases} \\
& I(x, y)= \begin{cases}0, & y \in[0,0.25], \\
\left(\frac{y-0.25}{0.5}\right)^{2}, & y \in[0.25,0.75], \\
1, & y \in[0.75,1] .\end{cases}
\end{aligned}
$$

Similarly, using solutions of the following functional equation (see [3, Proposition 3.6])

$$
f(\min (x+y, a))=f(x)+f(y),
$$

where $a>0$ is a fixed number, $x, y \in[0, a]$ and $f:[0, a] \rightarrow[0, \infty]$, one can obtain the following result.
Theorem 4.4. Let $T_{1}$ be a continuous t-norm, $T_{2}$ be a strict t-norm, $I:[0,1]^{2} \rightarrow[0,1]$ be a binary function and fix arbitrarily $x \in[0,1]$. If $u_{(I, x)}<v_{(I, x)}$ and the correspondingly generating t-norm $T_{m_{0}}$ of $T_{1}$ on the generating subinterval $\left[u_{(I, x)}, v_{(I, x)}\right]=\left[a_{m_{0}}, b_{m_{0}}\right]$ is nilpotent, then the following statements are equivalent:

(i) The triple of functions $T_{1}, T_{2}, I(x, \cdot)$ satisfies Eq. (D) for all $y, z \in[0,1]$.

(ii) $T_{1}$ admits the representation (3), and there exist continuous, strictly decreasing functions $t_{m_{0}}, t_{2}:[0,1] \rightarrow[0, \infty]$ with $t_{m_{0}}(1)=t_{2}(1)=0$, $t_{m_{0}}(0)<\infty, t_{2}(0)=\infty$, which are uniquely determined up to positive multiplicative constants, such that the correspondingly generating t-norm $T_{m_{0}}$ of $T_{1}$ on the generating subinterval $\left[a_{m_{0}}, b_{m_{0}}\right]$ and $T_{2}$ admit the representation (2) with $t_{m_{0}}, t_{2}$, respectively, and for the fixed above $x \in[0,1]$, the vertical section $I(x, \cdot)$ has the following form:

$$
I(x, y)= \begin{cases}0, & y \in\left[0, b_{m_{0}}\right), \\ 1, & y \in\left[b_{m_{0}}, 1\right] .\end{cases}
$$

Example 4.5. Assume that $T_{1}$ is a continuous t-norm given by the ordinal sum

$$
T_{1}=\left(\left\langle 0.25,0.75, T_{\mathbf{L K}}\right\rangle,\left\langle 0.75,1, T_{\mathbf{L K}}\right\rangle\right),
$$

thus

$$
T_{1}(x, y)=\left\{\begin{array}{r}
0.25+\max (x+y-1,0), \\
x, y \in[0.25,0.75], \\
0.75+\max (x+y-1.75,0), \\
x, y \in[0.75,1], \\
\min (x, y), \quad \text { otherwise. }
\end{array}\right.
$$

The set of idempotent elements for $T_{1}$ is the following:

$$
\operatorname{Idem}\left(T_{1}\right)=[0,0.25] \cup\{0.75,1\} .
$$

Let us assume that for any $x \in[0,1]$ we have $u_{(I, x)}=0.25$ and $v_{(I, x)}=0.75$. This means that $T_{m_{0}}=T_{\mathbf{L K}}$ is a nilpotent t-norm. One can easily check that the following function $I$ satisfies Eq. (D) with $T_{1}$ and any strict t-norm $T_{2}$ (for any $x, y, z \in[0,1])$ :

$$
I(x, y)=\left\{\begin{array}{ll}
0, & y \in[0,0.75), \\
1, & y \in(0.75,1],
\end{array} \quad x, y \in[0,1] .\right.
$$

Next, we can show the continuous solutions $I$ for Eq. (D). From previous theorems we see that there are only three such continuous solutions: $I=0$ or $I=1$ or $I$ has the form

$$
I(x, y)= \begin{cases}0, & y \in[0, a], \\ t_{2}^{-1}\left(c(x) t_{a}\left(\frac{y-a}{b-a}\right)\right), & y \in[a, b], \\ 1, & y \in[b, 1],\end{cases}
$$


for all $x, y \in[0,1]$, where $c:[0,1] \rightarrow(0, \infty)$ is a continuous function and the corresponding continuous Archimedean t-norm of $T_{1}$ on the subinterval $[a, b]$ is strict.

Example 4.6. Assume that $T_{1}$ is a continuous t-norm given by the ordinal sum

$$
T_{1}=\left(\left\langle 0.25,0.75, T_{\mathbf{P}}\right\rangle\right)
$$

thus

$$
T_{1}(x, y)=\left\{\begin{array}{cc}
0.25+2(x-0.25)(y-0.25), \\
\min (x, y), & \text { otherwise. }
\end{array}\right.
$$

The set of idempotent elements for $T_{1}$ is the following:

$$
\operatorname{Idem}\left(T_{1}\right)=[0,0.25] \cup[0.75,1] .
$$

Let us assume that for any $x \in[0,1]$ we have $u_{(I, x)}=0.25$ and $v_{(I, x)}=0.75$. This means that $T_{m_{0}}=T_{\mathbf{P}}$ is a strict t-norm and $t_{m_{0}}(x)=1-x$. One can easily check that the following continuous function $I$ satisfies Eq. (D) with $T_{1}$ and the nilpotent t-norm $T_{\mathbf{P}}$ (for any $x, y, z \in[0,1]$ ):

$$
I(x, y)= \begin{cases}0, & y \in[0,0.25], \\ (2(y-0.25))^{x+2}, & y \in[0.25,0.75], \\ 1, & y \in[0.75,1] .\end{cases}
$$

Corollary 4.7. If $T_{1}$ is a continuous t-norm and $\mathrm{T}_{2}$ is a strict $t$-norm, then there are no continuous solutions I of Eq. (D) which satisfy (I3).

From the above corollary it is obvious that we need to look for the solutions of Eq. (D) which are continuous except the point $(0,0)$. However, substituting $x=0$ and $z=0$ in Eq. (D), we can know that it follows that $I\left(0, T_{1}(y, 0)\right)=T_{2}(I(0, y), I(0,0))$ for all $y \in[0,1]$. That is, $I(0,0)=T(I(0, y), 1)$, which implies $I(0,0)=I(0, y)$. So it holds $I(0, y)=1$ for all $y \in[0,1]$. Thus we have proven the fact that it holds $I(0, y)=1$ for all $y \in[0,1]$ if the binary function $I$ satisfying Eq. (I3) is a solution of Eq. (D). On the other hand, applying the method of [16, Theorem 3.3], we can further know that there exist only solutions which are continuous except the vertical section $I(0, y)=1$ for $y \in[0, a]$.

Theorem 4.8. Let $T_{1}$ be a continuous t-norm, $T_{2}$ be a strict t-norm and I be a fuzzy implication continuous except the vertical section $I(0, y)=1$ for $y \in[0, a]$, where $a \in[0,1)$. Then the following statements are equivalent:

(i) The triple of functions $T_{1}, T_{2}, I$ satisfies Eq. (D) for all $x, y, z \in[0,1]$.

(ii) $T_{1}$ admits the representation (3), and there exist two constants $a<b \in[0,1]$ such that $u_{(I, x)}=a, v_{(I, x)}=b$ for all $x \in[0,1]$, and there exist continuous, strictly decreasing function $t_{a}, t_{2}:[0,1] \rightarrow[0, \infty]$ with $t_{a}(1)=t_{2}(1)=$
$0, t_{a}(0)=t_{2}(0)=\infty$, which are uniquely determined up to positive multiplicative constants, such that the correspondingly generating $t$-norm $T_{a}$ of $T_{1}$ on the generating subinterval $[a, b]$ and $T_{2}$ admit the representation (2) with $t_{a}$ and $t_{2}$, respectively, and there exists a continuous and increasing function $c:(0,1] \rightarrow$ $(0, \infty), c(0)=0$, uniquely determined up to a positive multiplicative constant depending on constants for $t_{a}$ and $t_{2}$, such that I has the form

$$
I(x, y)= \begin{cases}1, & x=0, y \in[0, a], \\ 0, & x \neq 0, y \in[0, a], \\ t_{2}^{-1}\left(c(x) t_{a}\left(\frac{y-a}{b-a}\right)\right), & y \in[a, b], \\ 1, & y \in[b, 1] .\end{cases}
$$

5. Solutions to Eq. (D) when $T_{1}$ is a continuous t-norm and $T_{2}$ is a nilpotent t-norm

In this section, we characterize the fuzzy implication $I$ satisfying Eq. (D) when $T_{1}$ is a continuous t-norms and $T_{2}$ is a nilpotent t-norm. From Section 3 it is enough to consider the case $u_{(I, x)}<v_{(I, x)}$. Now, using solutions of the following functional equation (see [3, Proposition 3.4])

$$
f(x+y)=\min (f(x)+f(y), b),
$$

where $b>0, x, y \in[0, \infty]$ and $f:[0, \infty] \rightarrow[0, b]$, one can obtain the following result.

Theorem 5.1. Let $T_{1}$ be a continuous t-norm, $T_{2}$ be a nilpotent $t$-norm, $I:[0,1]^{2} \rightarrow[0,1]$ be a binary function and fix $x \in[0,1]$. If $u_{(I, x)}<v_{(I, x)}$ and the correspondingly generating t-norm $T_{m_{0}}$ of $T_{1}$ on the generating subinterval $\left[u_{(I, x)}, v_{(I, x)}\right]=\left[a_{m_{0}}, b_{m_{0}}\right]$ is strict, then the following statements are equivalent:

(i) The triple of functions $T_{1}, T_{2}, I(x, \cdot)$ satisfies Eq. (D) for all $y, z \in[0,1]$.

(ii) $T_{1}$ admits the representation (3), and there exist continuous, strictly decreasing functions $t_{m_{0}}, t_{2}:[0,1] \rightarrow[0, \infty]$ with $t_{m_{0}}(1)=t_{2}(1)=0$, $t_{m_{0}}(0)=\infty, t_{2}(0)<\infty$, which are uniquely determined up to positive multiplicative constants, such that the correspondingly generating t-norm $T_{m_{0}}$ of $T_{1}$ on the generating subinterval $\left[a_{m_{0}}, b_{m_{0}}\right]$ and $T_{2}$ admit the representation (2) with $t_{m_{0}}, t_{2}$, respectively, and for the mentioned above $x \in[0,1]$, the vertical section $I(x, \cdot)$ has one of the following forms.

$$
\begin{gathered}
I(x, y)= \begin{cases}0, & y \in\left[0, a_{m_{0}}\right], \\
1, & y \in\left(a_{m_{0}}, 1\right],\end{cases} \\
I(x, y)= \begin{cases}0, & y \in\left[0, b_{m_{0}}\right), \\
1, & y \in\left[b_{m_{0}}, 1\right],\end{cases} \\
I(x, y)=\left\{\begin{array}{rr}
0, & y \in\left[0, a_{m_{0}}\right], \\
t_{2}^{-1}\left(\min \left(c_{x} t_{m_{0}}\left(\frac{y-a_{m_{0}}}{b_{m_{0}}-a_{m_{0}}}\right), t_{2}(0)\right)\right), & y \in\left[a_{m_{0}}, b_{m_{0}}\right], \\
1, & y \in\left[b_{m_{0}}, 1\right],
\end{array}\right.
\end{gathered}
$$


with a certain $c_{x} \in(0, \infty)$, uniquely determined up to a positive multiplicative constant depending on constants for $t_{m_{0}}$ and $t_{2}$.

Example 5.2. Let us consider the same continuous t-norm as in Example 3.5:

$$
T=\left(\left\langle 0.1,0.5, T_{\mathbf{P}}\right\rangle,\left\langle 0.7,0.9, T_{\mathbf{L K}}\right\rangle\right),
$$

Let us assume that for any $x \in[0,1]$ we have $u_{(I, x)}=0.1$ and $v_{(I, x)}=0.5$. This means that $T_{m_{0}}=T_{\mathbf{P}}$ is a strict t-norm and $t_{m_{0}}(x)=-\ln x$. Let us assume that $T_{2}(x, y)=T_{\mathbf{L K}}$ i.e., $t_{2}(x)=$ $1-x$. One can easily check that the following functions $I$ satisfies Eq. (D) (for any $x, y, z \in[0,1]$ ) with $T_{1}$ and $T_{2}$ :

$$
\begin{aligned}
& \text { - } I(x, y)= \begin{cases}0, & y \in[0,0.1], \\
1, & y \in(0.1,1],\end{cases} \\
& \text { - } I(x, y)= \begin{cases}0, & y \in[0,0.5), \\
1, & y \in[0.5,1],\end{cases} \\
& \text { - } I(x, y)= \begin{cases}0, & y \in[0,0.1], \\
1-\min \left(-\ln \left(\frac{y-0.1}{0.4}\right), 1\right), & y \in[0.1,0.5], \\
1, & y \in[0.5,1]\end{cases}
\end{aligned}
$$

Finally, using solutions of the following functional equation (see [5, Proposition 3])

$$
f(\min (x+y, a))=\min (f(x)+f(y), b),
$$

where $a, b>0, x, y \in[0, a]$ and $f:[0, a] \rightarrow[0, b]$, one can obtain the following result.

Theorem 5.3. Let $T_{1}$ be a continuous t-norm, $T_{2}$ be a nilpotent $t$-norm, $I:[0,1]^{2} \rightarrow[0,1]$ be a binary function and fix $x \in[0,1]$. If $u_{(I, x)}<v_{(I, x)}$ and the correspondingly generating t-norm $T_{m_{0}}$ of $T_{1}$ on the generating subinterval $\left[u_{(I, x)}, v_{(I, x)}\right]=\left[a_{m_{0}}, b_{m_{0}}\right]$ is nilpotent, then the following statements are equivalent:

(i) The triple of functions $T_{1}, T_{2}, I(x, \cdot)$ satisfies Eq. (D) for all $y, z \in[0,1]$.

(ii) $T_{1}$ admits the representation (3), and there exist continuous, strictly decreasing functions $t_{m_{0}}, t_{2}:[0,1] \rightarrow[0, \infty]$ with $t_{m_{0}}(1)=t_{2}(1)=0$, $t_{m_{0}}(0)<\infty, t_{2}(0)<\infty$, which are uniquely determined up to positive multiplicative constants, such that the correspondingly generating $t$-norm $T_{m_{0}}$ of $T_{1}$ on the generating subinterval $\left[a_{m_{0}}, b_{m_{0}}\right]$ and $T_{2}$ admit the representation (2) with $t_{m_{0}}, t_{2}$, respectively, and for the mentioned above $x \in[0,1]$, the vertical section $I(x, \cdot)$ has one of the following forms:

$$
\begin{gathered}
I(x, y)= \begin{cases}0, & y \in\left[0, b_{m_{0}}\right), \\
1, & y \in\left[b_{m_{0}}, 1\right],\end{cases} \\
I(x, y)=\left\{\begin{array}{rr}
0, & y \in\left[0, a_{m_{0}}\right], \\
t_{2}^{-1}\left(\min \left(c_{x} t_{m_{0}}\left(\frac{y-a_{m_{0}}}{b_{m_{0}}-a_{m_{0}}}\right), t_{2}(0)\right)\right), & y \in\left[a_{m_{0}}, b_{m_{0}}\right], \\
1, & y \in\left[b_{m_{0}}, 1\right],
\end{array}\right.
\end{gathered}
$$

with a certain $c_{x} \in\left[\frac{t_{2}(0)}{t_{\alpha}(0)}, \infty\right)$, uniquely determined up to a positive multiplicative constant depending on constants for $t_{m_{0}}$ and $t_{2}$.

Example 5.4. Let us consider the same continuous t-norm as earlier:

$$
T=\left(\left\langle 0.1,0.5, T_{\mathbf{P}}\right\rangle,\left\langle 0.7,0.9, T_{\mathbf{L K}}\right\rangle\right)
$$

Let us assume that for any $x \in[0,1]$ we have $u_{(I, x)}=0.7$ and $v_{(I, x)}=0.9$. This means that $T_{m_{0}}=T_{\mathbf{L K}}$ is a nilpotent t-norm and $t_{m_{0}}(x)=$ $1-x$. Let us assume that also $T_{2}(x, y)=T_{\mathbf{L K}}$ i.e., $t_{2}(x)=1-x$. One can easily check that the following function satisfy Eq. (D) (for any $x, y, z \in[0,1]$ ) with $T_{1}$ and $T_{2}$ :

$$
\begin{aligned}
I(x, y) & = \begin{cases}0, & y \in[0,0.1], \\
1-\min \left(1-\left(\frac{y-0.7}{0.2}\right), 1\right), & y \in[0.1,0.5], \\
1, & y \in[0.5,1],\end{cases} \\
& = \begin{cases}0, & y \in[0,0.1], \\
\max \left(\frac{y-0.7}{0.2}, 0\right), & y \in[0.1,0.5], \\
1, & y \in[0.5,1] .\end{cases}
\end{aligned}
$$

It should be noted that similarly as in Section 3, it is possible to obtain continuous solutions $I$ of (D), when $T_{2}$ is a nilpotent t-norm.

\section{Conclusion}

In this work, we continued to investigate the distributive equations of implication. It is our method that can still apply to the three other functional equations related closely to Eq. (D). It is in this sense that we make progress in solving the functional equation $I\left(x, S_{1}(y, z)\right)=$ $S_{2}(I(x, y), I(x, z))$ suggested by Baczyński and Balasubramaniam in [5]. Contrasted with the results obtained by Baczyński in [1], [2], [5], we can find many differences. For example, Eq. (D) has at most five kinds of the vertical section for all $x \in[0,1]$ when $T_{1}$ is a continuous and Archimedean t-norm, $T_{2}$ is a strict t-norm. While it has at most seven kinds of the vertical section for all $x \in[0,1]$ when $T_{1}$ is a continuous but not Archimedean t-norm, $T_{2}$ is a strict t-norm. In future works we will try to concentrate on the other cases of Eq. (D) that are not considered in this paper, for example, when both $T_{1}$ and $T_{2}$ are continuous but not Archimedean t-norms.

\section{Acknowledgements}

The work on this paper for the first author (Feng Qin) was supported by National Natural Science Foundation of China (No. 60904041), Jiangxi Natural Science Foundation (No. 2009GQS0055) and Scientific Research Foundation of Jiangxi Provincial Education Department (No. GJJ08160). Tel: +867913863755. 


\section{References}

[1] M. Baczyński, On a class of distributive fuzzy implications, Internat. J. Uncertainty, Fuzziness and Knowledge-Based Systems 9 (2001) 229-238.

[2] M. Baczyński, Contrapositive symmetry of distributive fuzzy implications, Internat. J. Uncertainty, Fuzziness and Knowledge-Based Systems 10 (2002) 135-147.

[3] M. Baczyński, On the distributivity of fuzzy implications over continuous and Archimedean triangular conorms, Fuzzy Sets and Systems 161 (2010) 1406-1419.

[4] M. Baczyński, B. Jayaram, Fuzzy implications (Studies in Fuzziness and Soft Computing, Vol. 231), Springer, Berlin 2008.

[5] M. Baczyński, B. Jayaram, On the distributivity of fuzzy implications over nilpotent or strict triangular conorms, IEEE Trans. Fuzzy Syst. 17 (2009) 590-603.

[6] J. Balasubramaniam, C.J.M Rao, On the distributivity of implication operators over T- and S-norms, IEEE Trans. Fuzzy Syst. 12 (2004) 194-198.

[7] W.E. Combs, J.E. Andrews, Combinatorial rule explosion eliminated by a fuzzy rule configuration, IEEE Trans. Fuzzy Syst. 6 (1998) $1-11$.

[8] W.E. Combs, Author's reply, IEEE Trans. Fuzzy Syst. 7 (1999) 371-373.

[9] W.E. Combs, Author's reply, IEEE Trans. Fuzzy Syst. 7 (1999) 477-478.

[10] S. Dick, A. Kandel, Comments on "Combinatorial rule explosion eliminated by a fuzzy rule configuration", IEEE Trans. Fuzzy Syst. 7 (1999) 475-477.

[11] J. Fodor, M. Roubens, Fuzzy Preference Modelling and Multicriteria Decision Support, Kluwer, Dordrecht, 1994.

[12] S. Gottwald, A Treatise on Many-Valued Logics, Research Studies, Baldock, 2001.

[13] E.P. Klement, R. Mesiar, E. Pap, Triangular Norms, Kluwer, Dordrecht, 2000.

[14] C.H. Ling, Representation of associative functions, Publ. Math. Debrecen 12 (1965) 189212.

[15] J.M. Mendel, Q. Liang, Comments on "Combinatorial rule explosion eliminated by a fuzzy rule configuration", IEEE Trans. Fuzzy Syst. 7 (1999) 369-371.

[16] F. Qin, Li Yang, Distributive equations of implications based on nilpotent triangular norms, Internat. J. Approx. Reason. 51 (2010) 984992.

[17] F. Qin, B. Zhao, The distributive equations for idempotent uninorms and nullnorms, Fuzzy Sets System 155 (2005) 446-458.

[18] D. Ruiz-Aguilera, J. Torrens, Distributivity of strong implications over conjunctive and dis- junctive uninorms, Kybernetika 42 (2005) 319336.

[19] D. Ruiz-Aguilera, J. Torrens, Distributivity of residual implications over conjunctive and disjunctive uninorms, Fuzzy Sets and Systems 158 (2007) 23-37.

[20] E. Trillas, C. Alsina, On the law $[p \wedge q \rightarrow r]=$ $[(p \rightarrow r) \vee(q \rightarrow r)]$ in fuzzy logic, IEEE Trans. Fuzzy Syst. 10 (2002) 84-88.

[21] I.B. Türksen, V. Kreinovich, R.R. Yager, A new class of fuzzy implications. Axioms of fuzzy implication revisted, Fuzzy Sets and Systems, 100 (1998) 267-272.

[22] L. Yang, F. Qin, Distributive equations based on fuzzy implications, in: IEEE International Conference on Fuzzy Systems, Korea 2009, pp. 560-563. 